\title{
USO DE PSICOFÁRMACOS ENTRE IDOSOS USUÁRIOS DO SISTEMA ÚNICO DE SAÚDE E DO PLANO DE SAÚDE SUPLEMENTAR
}

\author{
Graziela Maria Ferraz de Almeida \\ Carlos Alberto Lazarini² \\ Isabel Cristina Aparecida Stefano ${ }^{3}$ \\ Elaine Cristina Salzedas Muniz ${ }^{4}$ \\ Vanessa Cliveralo Bertassi Panes ${ }^{5}$ \\ Maria José Sanches Marin ${ }^{6}$
}

resumo

Objetivo: Comparar o uso de psicofármacos entre idosos que utilizam o Sistema Único de Saúde (SUS) e aqueles que utilizam o Plano de Saúde Suplementar (PSS). Método: Estudo quantitativo

1 Graduada em Enfermagem. Residência Multiprofissional em Saúde do Adulto e do Idoso pela Universidade Estadual Paulista (UNESP) de Botucatu. E-mail: ga_graziela@hotmail.com.

2 Graduado em Farmacologia. Doutor em Farmacologia. Docente da Faculdade de Medicina de Marília. E-mail: carlos.lazarini@gmail.com.

3 Graduada em Enfermagem. Mestre em Saúde e Envelhecimento. Enfermeira no Departamento Regional de Saúde de Marilia (DRS IX). E-mail: belstefano@hotmail.com.

4 Graduada em Enfermagem. Mestre em Saúde e Envelhecimento. E-mail: elacris@terra.com.br.

5 Graduada em Enfermagem. Doutora em Enfermagem. Docente do Curso de Medicina da Fundação Educacional do Município de Assis. E-mail: bertassi@hotmail.com.

6 Graduada em Enfermagem. Doutora em Enfermagem. Docente do Curso de Enfermagem da Faculdade de Medicina de Marília. E-mail: marnadia@terra.com.br. 
e retrospectivo realizado a partir de dois bancos de dados sendo: 239 idosos usuários do PSS e 114 usuários da Estratégia Saúde da Família (ESF). As análises estatísticas foram realizadas por meio do software Statistical Package for Social Sciences (SPSS) versão 17.0 para Windows. As análises inferenciais foram realizadas por meio do teste de qui-quadrado de Pearson e a extensão do teste exato de Fisher. Resultados: Entre os usuários do SUS, 53\% têm de 60 a 69 anos e 13\% mais de 80 anos; entre os usuários do PSS, 37\% têm de 60 a 69 anos e 24\% mais de 80 anos. Entre os usuários do SUS há maior prevalência de pele negra ou parda, baixa escolaridade e classe socioeconômica mais baixa. Encontrou-se que 68\% dos usuários do SUS e 60\% daqueles que utilizam o PSS não fazem uso de psicofármacos. Os psicofármacos mais prescritos são os antidepressivos. A amitriptilina, fluoxetina, diazepam, clonazepam e alprazolam, que são fármacos contidos em medicamentos de menor custo, porém, considerados potencialmente inapropriados para idosos, foram prescritos principalmente aos usuários do SUS. Conclusão: Os usuários do SUS e do PSS apresentam características sociodemográficas diferenciadas. Os usuários do SUS utilizam maior quantidade de medicamentos potencialmente inapropriados, enquanto aos usuários do plano de saúde são prescritos medicamentos tecnologicamente mais avançados e com menor possibilidade de desencadear efeitos indesejáveis.

palavras-chave

Idoso. Psicotrópicos. Sistema Único de Saúde. Planos de Pré-pagamento em Saúde.

$\mathrm{Na}$ atualidade, o aumento na expectativa de vida da população leva a uma sociedade com mais idosos e, com isso, maior demanda dessa parcela da população aos serviços de saúde em geral (OLIVEIRA; MENEZES, 2014). O Estatuto do Idoso define pessoa idosa como aquela com 60 anos ou mais, mesmo compreendendo que o critério cronológico não é o único a ser considerado quando se propõe a tal definição de forma mais ampliada, uma vez que são muitos os fatores que interferem nesse processo, fazendo com que as pessoas envelheçam mais precocemente ou mais tardiamente (BRASIL, 2013a). 
De qualquer forma, há o reconhecimento de que, com o envelhecimento, a funcionalidade do organismo diminui. Pelo enfoque biológico, o envelhecer é um processo dinâmico e crescente, com perda gradual da eficácia da adaptação da pessoa no meio ambiente, tornando o idoso vulnerável a processos patológicos, dentre os quais, encontram-se os transtornos mentais (BOTTAN, 2013).

O transtorno mental é percebido por mudanças desconfortantes do comportamento, do pensamento e percepção, o que prejudica a capacidade de interagir socialmente, conviver e ter autonomia. O portador de transtorno mental apresenta sintomas de ansiedade e depressão como irritabilidade, insônia, esquecimento, dificuldade de concentração, fadigas e queixas somáticas (VIDAL et al., 2013). A maioria não identifica a presença do transtorno, mas percebe situações de sofrimento. De qualquer forma, ocorre redução da qualidade vida das pessoas (CLEMENTE; LOYOLA FILHO; FIRMO, 2011; ROCHA et al., 2012). Como os idosos têm maior tendência a apresentar número maior de doenças, ficam expostos ao maior consumo de medicação e, por consequência, aos riscos do uso inadequado. Os idosos são mais vulneráveis aos efeitos adversos de medicamentos, além de utilizarem simultaneamente vários fármacos e, por vezes, administrados erroneamente (PAULA; BOCHNER; MONTILLA, 2012).

Estudo que analisou as condutas adotadas na Estratégia Saúde da Família (ESF), com ênfase nos aspectos relacionados à saúde mental, identificou a prevalência de transtornos mentais por meio da aplicação do Self-Response Questionnaire (SRQ-20) em 219 idosos, e constatou que houve apenas oito encaminhamentos para os serviços especializados em saúde mental no período de um ano. Os medicamentos psiquiátricos mais prescritos foram os ansiolíticos (55\%) e antidepressivos (29,7\%). Escores do SRQ-20 acima de sete estiveram presentes em $19,6 \%$ dos idosos. Os autores concluíram que grande parte dos problemas não é detectada e nem sempre as condutas medicamentosas (ou não) são adequadas (ONOFRI JÚNIOR; MARTINS; MARIN, 2016).

Os psicofármacos são substâncias químicas que agem sobre situações psicológicas, modificando o estado mental, e incluem as classes dos antidepressivos, alucinógenos, ansiolíticos e antipsicóticos. O uso de psicofármacos por idosos, nas últimas décadas, virou um assunto de debate na farmacoepidemiologia, pois vem ocorrendo um aumento expressivo do consumo destes produtos (NOIA et al., 2012). Alguns medicamentos e, principalmente, algumas classes dos psicofármacos, são considerados inapropriados para idosos, devido ao risco de efeitos adversos e à falta de eficácia terapêutica (CASSONI et al., 2014), ou seja, é considerado inadequado para o idoso quando os potenciais riscos são superiores aos benefícios (OLIVEIRA; CERDEIRA; BARROS, 2017). 
Frente à relevância da prescrição que atenda às especificidades farmacodinâmicas e farmacocinéticas dos idosos, em 1991, foram criados, por Beers-Fick, critérios para prevenir as prescrições inadequadas de fármacos para idosos com 65 anos ou mais. Além dos critérios de Beers-Fick, existem outros com a finalidade de detectar medicamentos inapropriados, como os critérios STOPP/ START, da Irlanda e Reino Unido (BUENO; ALMEIDA; ROCHA, 2016) e os critérios PRISCUS, da Alemanha (HOLT; SCHMIEDL; THÜRMANN, 2010).

No Brasil, foi constatado por meio de revisão da literatura que os critérios de Beers-Fick ainda são os mais utilizados, embora os critérios PRISCUS ofereçam alguns avanços, uma vez que ampliam a lista de medicamentos e proporcionam observações para a prática clínica e opções terapêuticas (GORZONI; FABBRI; PIRES, 2012). O contexto que envolve o uso de psicofármacos por idosos reveste-se de grande complexidade e remete a reflexões quanto aos modelos de atenção à saúde que os idosos utilizam, visto que, eles podem ser atendidos tanto pela Atenção Básica à Saúde, enquanto porta de entrada para os diferentes níveis de atenção oferecidos pelo Sistema Único de Saúde (SUS), recebendo inicialmente uma atenção de generalista, assim como, podem contar com o Plano de Saúde Suplementar (PSS), que oferece maior flexibilidade para o acesso a serviços específicos, como é o caso do atendimento por especialistas.

Vale ressaltar que, no Brasil, o SUS tem o compromisso de ofertar atendimento a toda população, de maneira igualitária e equânime. Apesar de diversas iniciativas do governo federal para a construção e indução de processos que ampliem e assegurem o acesso e melhorem a qualidade da atenção, a população está procurando cada vez mais serviços privados e convênios, devido a precariedade na infraestrutura, bem como à falta de profissionais em quantidade e adequadamente qualificados para o atendimento das demandas da população (DILÉLIO et al., 2014).

Estudo sobre o uso de medicamentos em idosos que utilizam o PSS revelou que 97,1\% utilizavam algum medicamento; com média de 5,8 medicamentos/ idoso, sendo 62,8\% submetidos à polifarmácia; 11,7\% utilizavam medicamentos inapropriados para idosos e 51,0\% deles apresentavam média adesão aos medicamentos, sugerindo um modelo de atenção pautado no tratamento de doenças e na farmacoterapia, embora esses idosos tenham melhor nível de escolaridade e poder aquisitivo. Do total de medicamentos utilizados 18,7\% foram para o sistema nervoso (MUNIZ et al., 2017).

Uma análise de prontuários de idosos que receberam tratamento psicoterápico revelou número expressivo de medicação psicotrópica, inclusive com a utilização de mais de um psicofármaco por idoso, o que para os autores reforça os apontamentos existentes na literatura sobre o aumento da medicação 
psicofarmacológica (SILVA; HERZOG, 2015). Frente ao aumento no uso de psicofármacos por idosos, a relevância do uso apropriado de medicamentos entre eles e considerando que pode haver diferenças entre os psicofármacos prescritos para usuários do SUS e do PSS, o presente estudo tem como objetivo comparar o uso de psicofármacos entre idosos que utilizam o SUS e aqueles que utilizam o PSS.

\section{Método}

Trata-se de um estudo quantitativo, retrospectivo, envolvendo o uso de psicofármacos por idosos residentes no município de Marília, a partir de dois bancos de dados, um com idosos usuários de PSS, e outro com idosos usuários do SUS. A cidade de Marília está situada no Centro Oeste Paulista do estado de São Paulo, com 216.745 habitantes (IBGE, 2017), sendo município de referência estadual em saúde para mais 62 municípios. Atualmente, possui 34 unidades da ESF e 12 Unidades Básicas de Saúde (UBS). O índice de envelhecimento populacional é de $86,5 \%$ e a população com 60 anos ou mais gira em torno de $15,3 \%$. Os usuários de plano de saúde de Marília somam 50.578, ou seja, 22,5\% da população total, desses, $16,75 \%$ tem idade superior a 58 anos, segundo dados oferecidos pela operadora do plano de saúde. A ESF atende em torno de 110.000 pessoas, ou seja, $50 \%$ da população de Marília.

Os bancos de dados utilizados apresentam as seguintes características:

Banco de dados I - Idosos usuários do PSS. O estudo contou com população idosa com 60 anos ou mais, homens e mulheres, que não estavam asiladas e nem hospitalizadas, usuários de um PSS de maior abrangência no município de Marília - SP. O cálculo amostral foi realizado a partir do número de idosos usuários do plano de saúde de 8.474 pessoas, da prevalência de uso de medicamentos de $80 \%$, da margem de erro de $5 \%$ e nível de confiança de $95 \%$, resultando em 239 indivíduos $(\mathrm{N}=239)$.

Banco de dados II - Idosos usuários do SUS. Para o estudo, foi realizada coleta de dados em oito unidades da ESF, sendo duas em cada região, a fim de obter representatividade da população de sua abrangência. Neste banco, foram incluídos idosos com mais de 60 anos, que tiveram consulta médica na semana anterior a da coleta de dados e que receberam prescrição de medicamentos. Foi considerada para o cálculo amostral, a população estimada de idosos em $13 \%$ da população atendida pela ESF, a prevalência de $50 \%$ de uso de medicamentos, margem de erro de $10 \%$ e nível de confiança de $95 \%$. Foi acrescido ao valor calculado, $20 \%$ para cobrir possíveis perdas ou recusas. 
Isto resultou em uma amostra de 114 usuários $(\mathrm{N}=114)$. Os idosos que não foram encontrados após três visitas domiciliares foram excluídos da pesquisa.

De acordo com o objetivo do presente estudo, foram utilizados os dados sociodemográficos e epidemiológicos, como: idade, sexo, estado civil, cor da pele, escolaridade, ocupação e classe social, de acordo com o Critério de Classificação Econômica Brasil (ABEP, 2014), além do número e tipo de psicofármacos utilizados. Para a comparação entre os dados, a natureza do prestador de serviços foi considerada como variável independente. As demais variáveis foram consideradas como dependentes. As análises estatísticas relativas às comparações entre os dois bancos de dados foram realizadas por meio do software Statistical Package for Social Sciences (SPSS) versão 17.0 para Windows. As análises inferenciais foram realizadas por meio do Teste de Qui-quadrado de Pearson e a extensão do teste Exato de Fisher. Em todas as conclusões obtidas pelas análises inferenciais foi utilizado o nível de significância $\alpha$ igual a 5\% $(p \leq 0,05)$. O presente estudo foi previamente aprovado pelo Comitê de Ética com Seres Humanos da Faculdade de Medicina de Marília, sob Protocolo n ${ }^{\circ}$ 105.049, de 10/10/2016.

\section{Resultados}

A Tabela 1 apresenta os dados sociodemográficos. A análise estatística mostra diferenças significantes ao se comparar os dois prestadores de serviços. Entre os idosos usuários do PSS, há predomínio da faixa etária entre 80 anos ou mais $(\mathrm{p}=0,005)$, do sexo feminino $(\mathrm{p}=0,001)$, das cores da pele branca e amarela ( $p=0,001)$, das classes socioeconômicas B e D ( $p=0,001)$ e da escolaridade acima de quatro anos $(\mathrm{p}=0,001)$. Já os usuários do SUS apresentaram predomínio da faixa etária entre 60 e 69 anos ( $\mathrm{p}=0,005)$, da cor da pele parda/ negra $(p=0,001)$, da classe econômica $C(p=0,001)$ e da escolaridade até quatro anos $(p=0,001)$. Quanto ao estado civil não houve diferença estatisticamente significante entre os dois prestadores de serviço $(p=553)$. 
Tabela 1 - Distribuição dos idosos usuários do Plano de Saúde Suplementar e usuários

Variáveis

\section{Usuários do SUS Usuários do PSS}

\begin{tabular}{crcc} 
& $(\mathrm{N}=114)$ & \multicolumn{2}{c}{$(\mathrm{N}=239)$} \\
\hline $\mathrm{N}$ & $\%$ & $\mathrm{~N}$ & $\%$
\end{tabular}

\section{Idade}

$60-69$

$61 \quad 53,5 \quad 90 \quad 37,7$

$70-79$

39

$34,2 \quad 91$

38,1

$0,005^{\star}$

$\geq 80$

14

12,3

58

24,2

Sexo

Masculino

43

$37,7 \quad 50$

21,0

Feminino

71

$62,2 \quad 189$

79,0

$0,001^{*}$

Cor da pele

Branca

Parda/Negra

Amarela

\section{Estado civil}

Solteiro, viúvo,

separado, divorciado

Casado/união estável

\section{Classe econômica}

Classe A

Classe B

Classe C

Classe D

\section{Escolaridade}

$\begin{array}{lccccc}0 \text { a } 4 \text { anos } & 105 & 92,1 & 59 & 24,7 & \\ +4 \text { anos } & 9 & 7,9 & 179 & 74,9 & 0,001^{*} \\ \text { Não respondeu } & 0 & 0,0 & 1 & 0,4 & \end{array}$

Fonte: Elaborada pelos autores.

* p $\leq 0,05$ (Teste do Qui-quadrado de Pearson)

$\begin{array}{lllll}66 & 57,9 & 193 & 80,7 & \\ 43 & 37,7 & 18 & 7,5 & 0,001^{*} \\ 5 & 4,4 & 28 & 11,8\end{array}$

58

50,9

112

46,9

0,553

$\begin{array}{llll}56 & 49,1 & 127 & 53,1\end{array}$ 
Entre os idosos entrevistados, 95 (39,7\%) daqueles que utilizam o PSS e $36(31,6 \%)$ usuários do SUS fazem uso de psicofármacos, sendo que a maioria utiliza apenas um. Na análise comparativa quanto ao uso dessa classe de medicamentos, usuários do PSS utilizam psicofármacos em maior quantidade quando comparados aos usuários do SUS $(\mathrm{p}=0,015)$. Em relação ao número de medicamentos que utilizam, a análise estatística não mostrou diferenças estatisticamente significantes. A comparação entre as classes de psicofármacos mostrou diferenças significantes, sendo que, os usuários do PSS utilizam mais ansiolíticos quando comparados aos do SUS $(\mathrm{p}=0,035)$ (Tabela 2$)$.

Tabela 2 - Distribuição dos idosos usuários do Plano de Saúde Suplementar e usuários do Sistema Único de Saúde, de acordo com o uso, quantidade e classe de psicofármacos. Marília, 2017.

\begin{tabular}{|c|c|c|c|c|c|}
\hline \multirow{2}{*}{ Variáveis } & \multicolumn{2}{|c|}{ Usuários do SUS } & \multicolumn{2}{|c|}{ Usuários do PSS } & \multirow{2}{*}{$p$} \\
\hline & $\mathrm{N}$ & $\%$ & $\mathrm{~N}$ & $\%$ & \\
\hline \multicolumn{6}{|l|}{ Uso de psicofármacos } \\
\hline Sim & 36 & 31,6 & 95 & 39,7 & $0,015^{\star}$ \\
\hline Não & 78 & 68,4 & 144 & 60,3 & \\
\hline \multicolumn{6}{|c|}{ Quantidade de psicofármacos } \\
\hline 1 & 24 & 66,7 & 61 & 64,2 & 0,479 \\
\hline 2 & 7 & 19,4 & 26 & 27,4 & \\
\hline 3 & 3 & 8,3 & 6 & 6,3 & \\
\hline 4 & 1 & 2,8 & 0 & 0,0 & \\
\hline 5 ou mais & 1 & 2,8 & 2 & 2,0 & \\
\hline \multicolumn{6}{|c|}{ Classe dos psicofármacos } \\
\hline Antidepressivos & 32 & 57,1 & 67 & 47,2 & 0,207 \\
\hline Ansiolíticos & 15 & 26,8 & 61 & 43,0 & $0,035^{\star}$ \\
\hline Antipsicóticos & 7 & 12,5 & 9 & 6,3 & 0,152 \\
\hline Estabilizadores de humor & 1 & 1,8 & 2 & 1,4 & 0,612 \\
\hline Antidemenciais & 1 & 1,8 & 3 & 2,1 & 0,682 \\
\hline
\end{tabular}

Fonte: Elaborada pelos autores.

* p $\leq 0,05$ (Teste do Qui-quadrado de Pearson) 
Quanto aos medicamentos psicofármacos utilizados (Tabela 3), a análise estatística mostrou associação positiva entre alguns tipos de antidepressivos e a natureza do prestador de serviços. Os usuários do SUS utilizam a amitriptilina $(p=0,001)$ e a fluoxetina $(p=0,020)$ em maior proporção, enquanto os usuários do PSS utilizam outros tipos de antidepressivos, como o escitalopram $(\mathrm{p}=0,038)$ e a paroxetina $(\mathrm{p}=0,025)$. Referindo-se aos antipsicóticos, os usuários do SUS fazem uso de clorpromazina e haloperidol em maior proporção, embora a análise estatística não tenha mostrado diferença significante. Os usuários do PSS utilizam outros medicamentos antipsicóticos, como: risperidona, carbamazepina, levomepromazina, olanzapina e quetiapina, também sem diferenças significantes.

Na classe de ansiolíticos, a análise estatística mostrou associação positiva, sendo que, os usuários do SUS utilizam em maior proporção diazepam $(\mathrm{p}=0,000)$ e nitrazepam $(\mathrm{p}=0,037)$. $\mathrm{O}$ antidemencial utilizado por três usuários do PSS foi galantamina, enquanto um usuário do SUS utiliza rivastigmina. Ainda, tanto os usuários do SUS quanto os do PSS fazem uso de estabilizadores do humor.

Tabela 3 - Distribuição dos idosos usuários do Plano de Saúde Suplementar e usuários do Sistema Único de Saúde, de acordo com os psicofármacos utilizados. Marília, 2017.

\begin{tabular}{|c|c|c|c|c|c|}
\hline \multirow{2}{*}{ Medicamentos } & \multicolumn{2}{|c|}{ Usuários do SUS } & \multicolumn{2}{|c|}{ Usuários do PSS } & \multirow{2}{*}{$p$} \\
\hline & $\mathrm{N}$ & $\%$ & $\mathrm{~N}$ & $\%$ & \\
\hline \multicolumn{6}{|l|}{ Antidepressivos } \\
\hline Amitriptilina & 12 & 37,5 & 5 & 7,5 & $0,001^{*}$ \\
\hline Citalopram & 1 & 3,1 & 8 & 11,4 & 0,145 \\
\hline Escitalopram & 0 & 0,0 & 8 & 11,4 & $0,038 \#$ \\
\hline Fluoxetina & 12 & 37,5 & 11 & 16,4 & $0,020^{*}$ \\
\hline Nortriptilina & 1 & 3,1 & 3 & 4,5 & 0,611 \\
\hline Paroxetina & 0 & 0,0 & 9 & 13,4 & $0,025 \#$ \\
\hline Sertralina & 3 & 9,4 & 7 & 10,5 & 0,588 \\
\hline Venlafaxina & 0 & 0,0 & 4 & 6,0 & 0,301 \\
\hline Outros & 3 & 9,4 & 12 & 17,9 & 0,213 \\
\hline
\end{tabular}




\begin{tabular}{lccccc}
\hline \multirow{2}{*}{ Medicamentos } & \multicolumn{2}{l}{ Usuários do SUS } & \multicolumn{2}{c}{ Usuários do PSS } & p \\
\cline { 2 - 4 } & $\mathbf{N}$ & $\%$ & $\mathbf{N}$ & $\%$ & \\
\hline Ansiolíticos & & & & & \\
Alprazolam & 0 & 0 & 10 & 16,4 & 0,094 \\
Bromazepam & 0 & 0 & 11 & 18,0 & 0,073 \\
Clonazepam & 6 & 40,0 & 29 & 47,5 & 0,409 \\
Diazepam & 6 & 40,0 & 0 & 0,0 & $0,000 \#$ \\
Nitrazepam & 2 & 13,3 & 0 & 0,0 & $0,037 \#$ \\
Passiflora & 1 & 6,7 & 2 & 3,3 & 0,482 \\
Valeriana & 0 & 0,0 & 3 & 4,9 & 0,512 \\
Outros & 0 & 0,0 & 6 & 9,8 & 0,254
\end{tabular}

\section{Antipsicóticos}

Clorpromazina

Haloperidol

Risperidona

Outros (carbamazepina,

levomepromazina, olanzapina, quetiapina)

\section{Estabilizadores do humor}

Carbonato de lítio

Divalproato de sódio

\section{Antidemenciais}

$\begin{array}{llllll}\text { Galantamina } & 0 & 0 & 3 & 100,0 & 0,250 \\ \text { Rivastigmina } & 1 & 100,0 & 0 & 0\end{array}$

Fonte: Elaborada pelos autores.

* $\mathrm{p} \leq 0,05$ (Teste do Qui-quadrado de Pearson)

\# p $\leq 0,05$ (Teste Exato de Fisher) 
Na comparação dos dados referentes ao uso de psicofármacos entre idosos usuários do SUS e PSS, destacam-se, nesta pesquisa, diferenças estatisticamente significantes na maioria dos os aspectos sociodemográficos analisados. O perfil mais envelhecido foi observado entre idosos beneficiários do PSS quando comparado àqueles sem plano de saúde. Contudo, é descrito que, entre os idosos que possuem PSS no país, a maioria encontra-se na faixa de idade entre 60-69 anos (ANS, 2016).

Assim como no presente estudo, dados do IBGE apontam para a feminilização da velhice (IBGE, 2012, 2013) e que o envelhecimento é também uma questão de gênero, uma vez que as mulheres se expõem menos ao risco de adoecimento e morte (SANTOS et al., 2013). Quando se trata de idosos que utilizam plano de saúde, para cada 100 homens com 60 anos ou mais, existem 143 mulheres (ANS, 2016). Os dados com os usuários do PSS revelaram que as mulheres se encontram mais disponíveis para a participação na pesquisa e, portanto, colaboraram mais que os homens.

Quanto ao Critério de Classificação Econômica Brasil (ABEP, 2014), que tem a função de estimar o poder de compra das pessoas e famílias urbanas, tem-se a indicação de que, principalmente os idosos que utilizam o PSS, contam com recursos que lhes permitem a aquisição de bens de consumo e uma vida com mais conforto. Referindo-se ao atendimento médico ambulatorial no Brasil, foi encontrado que o SUS atendeu $53,6 \%$ da população, os PSS 33,9\% e os serviços privados $12,5 \%$. As classes A e B usaram $59,4 \%$ de atendimento em convênio e $15,5 \%$ em particular e as classes D e E utilizaram em $85,1 \%$ o SUS. Além disso, indivíduos que se autodeclararam brancos e vivem com companheiro, utilizam mais os serviços de saúde (DILÉLIO et al., 2014).

Dados da Agência Nacional de Saúde Suplementar (ANS, 2012), mostram que, quando a renda domiciliar per capita é inferior a $1 / 4$ do salário mínimo, 0 acesso ao plano de saúde é bastante diminuto, tanto na população total como na de idosos. Sem dúvida, os maiores níveis de renda permitem aquisição de melhores serviços de acompanhamento, equipamentos de apoio e inserção social mais ativa (IBGE, 2013).

A Pesquisa Nacional de Amostras de Domicílios, de 2009 (PNAD, 2010) revelou que cerca da metade dos idosos tem menos de quatro anos de estudo. Diferenças expressivas foram observadas entre os idosos com acesso ao plano de saúde, uma vez que $18 \%$ deles possuem ensino superior e $19 \%$, o ensino médio. No presente estudo, verificou-se que a maioria dos usuários do plano de saúde relatou mais de quatro anos de estudo. 
Tem-se constatado que idosos com poucos anos de estudo ou analfabetos podem ter dificuldade na compreensão das prescrições e apresentar menor adesão farmacoterapêutica (TAVARES et al., 2016). A adesão ao tratamento medicamentoso também pode ser afetada pelo alto custo, maior sensibilidade dos idosos em apresentarem efeitos adversos, esquecimento (às vezes ou sempre), uso de várias medicações, cronicidade de doenças, presença de sintomas depressivos e falta de uma prescrição apropriada (MARIN et al., 2008; TAVARES et al., 2016).

No presente estudo, a maioria dos psicofármacos utilizados pertence à classe dos antidepressivos. Os usuários do SUS utilizam em maior proporção a amitriptilina e a fluoxetina, enquanto os usuários do PSS utilizam outros tipos de antidepressivos, como o escitalopram e a paroxetina. A menor diversidade de prescrição aos usuários do SUS pode estar associada à disponibilidade desses medicamentos no serviço público. Nesse sentido, tanto a amitriptilina quanto a fluoxetina estão presentes na Relação Nacional de Medicamentos Essenciais (RENAME) (BRASIL, 2015), a qual serve de base para a aquisição de medicamentos a serem disponibilizados pelas ESFs (BRASIL, 2015).

Em contrapartida, tanto escitalopram quanto paroxetina não constam nessa relação. Além disso, esses dois últimos possuem custo maior que os anteriores, muitas vezes impossibilitando sua aquisição pelos usuários do SUS. Ao se avaliar a eficácia e a segurança dos inibidores seletivos da receptação da serotonina (ISRS), uma revisão sistemática envolvendo 15 ensaios clínicos que abordaram o uso de antidepressivos em idosos mostrou que a sertralina, a paroxetina e a duloxetina são os ISRS mais eficazes no tratamento da depressão maior em idosos (THORLUND et al., 2015). Nesse sentido, as prescrições mais adequadas desses antidepressivos estão acontecendo no cenário do PSS.

Da mesma forma, ao considerar a lista de Medicamentos Potencialmente Inapropriados (MPI) para idosos, tanto considerando o critério de Beers-Fick como os de PRISCUS, a amitriptilina e a fluoxetina prescritas com maior frequência aos usuários do SUS, enquadram-se como MPI (AGS, 2015; HOLT; SCHMIEDL; THÜRMANN, 2010).

Outro ponto que não pode ser ignorado é o fato de que alguns profissionais seguem a orientação do Ministério da Saúde e prescrevem antidepressivos tricíclicos (amitriptilina) para o tratamento paliativo da dor em idosos (BRASIL, 2013b). Como o presente trabalho não avaliou as prescrições, caso isso tenha ocorrido, o mesmo pode ser entendido como uma variável de confusão.

Referindo-se aos antipsicóticos, os usuários do SUS tendem a fazer uso de clorpromazina e haloperidol e, os usuários do PSS de outros medicamentos como carbamazepina, levomepromazina, olanzapina e quetiapina. Vale 
ressaltar que, com exceção da levomepromazina, todos os demais antipsicóticos estão presentes na RENAME. Tanto a clorpromazina quanto o haloperidol são classificados com antipsicóticos típicos e são bastante efetivos para redução dos sintomas positivos da esquizofrenia. Já a olanzapina e a quetiapina são classificados como atípicos e melhoram tanto sintomas positivos quanto negativos. Nesse sentido, os mais prescritos pela PSS são mais eficazes no tratamento da esquizofrenia. Considerando que tanto típicos quanto atípicos estão presentes na RENAME, o que pode justificar a menor prescrição dos antipsicóticos atípicos pelo SUS pode ser o alto custo dos mesmos para o sistema de saúde, uma vez que mesmo constando na lista de medicamentos essenciais, nem sempre os medicamentos com custo maior estão disponíveis para a distribuição gratuita, o que limita o seu uso pelo idoso com menor poder aquisitivo (COLET; BORGES; AMADOR, 2016).

Referindo-se ainda aos antipsicóticos, a clorpromazina é considerada como MPI pelos critérios de Beers-Fick e de PRISCUS, enquanto a levomepromazina e olanzapina, quando em dosagem acima de $10 \mathrm{mg}$, apenas pelos critérios de PRISCUS. Da classe dos ansiolíticos, os usuários do PPS utilizam em maior proporção o alprazolam, bromazepam e clonazepam (BRASIL, 2015), sendo esse último também utilizado por usuários do SUS, além do diazepam (BRASIL, 2015), o que não ocorre com os usuários do PSS. Novamente, a justificativa para esta diferença de prescrição entre esses dois cenários pode estar associada ao fato do SUS adquirir medicamentos constantes na RENAME. Nesse sentido, tanto o clonazepam quanto o diazepam constam na relação do RENAME. No entanto, esses dois últimos medicamentos são considerados MPI para idosos, assim como o alprazolam (FICK et al., 2003).

Em relação aos antidemenciais, o utilizado por três usuários do PSS foi a galantamina e pelo usuário do SUS foi a rivastigmina (BRASIL, 2015). Ao se avaliar os dados das compras realizadas pelo Ministério do Planejamento, Orçamento e Gestão, entre 2008 e 2013, foi encontrado que o governo federal adquiriu mais rivastigmina que galantamina, numa relação de 5,6 vezes (COSTA et al., 2015). Esse fato pode justificar a prescrição da rivastigmina observada nos usuários do SUS. Além disso, em pesquisa de mercado, foi constatado que a galantamina tem preço menor, em comparação com a rivastigmina. No que se refere a eficácia terapêutica, dados da literatura mostram que esses medicamentos são prescritos para o tratamento da doença de Alzheimer, sendo parcialmente efetivos no enfrentamento dos sintomas cognitivos da doença (CARVALHO; CRISTINO; LIMBERGER, 2018).

Nessa perspectiva, na caracterização do uso de medicamento por diferentes classes socioeconômicas foi constatado que a classe A adquire seus 
medicamentos em estabelecimentos privados, sendo que o custo mais alto foi atribuído aos antipsicóticos. As classes C e E utilizam os medicamentos que são fornecidos pelos serviços públicos (COLET; BORGES; AMADOR, 2016).

Estudo que avaliou as classes de psicofármacos inapropriados para idosos, pela classificação STOPP/START e pelos critérios de Beers-Fick, mostrou que 50,4\% foram de antidepressivos, 20,4\% de ansiolíticos, 16,1\% de antiepiléticos e $21,4 \%$ de antipsicóticos. A fluoxetina foi o psicofármaco com maior prevalência em prescrição (29,4\%), seguida pela amitriptilina, diazepam e clonazepam (BUENO; ALMEIDA; ROCHA, 2016).

É importante considerar que a maioria dos psicofármacos são classificados como inapropriados devido às propriedades sedativas, que podem aumentar a ocorrência de síncopes (FICK et al., 2003) e quedas (ALVARENGA et al., 2015). Pode ser considerado como limitações do estudo aquelas que são próprias dos estudos transversais, ou seja, os bancos de dados utilizados retratam o uso de medicamentos entre os idosos em um único momento, além disso, o estudo é restrito a um único município. Mesmo assim, reconhece-se sua relevância, visto que, em especial revela as diferenças do tratamento farmacológico indicado na rede pública e na rede privada.

\section{Conclusões}

O presente estudo possibilitou a comparação de dois grupos de idosos quanto ao uso de psicofármacos, sendo um deles usuário do SUS e outro de PSS. Nos dados sociodemográficos, encontraram-se diferenças significantes em todos os aspectos analisados, exceto quanto ao estado conjugal. Quanto aos psicofármacos utilizados, a maioria pertence à classe dos antidepressivos, sendo que as prescrições para os usuários do PSS indicam o uso de medicamentos de melhor eficácia clínica. O mesmo foi observado no uso dos antipsicóticos. Quanto aos ansiolíticos, os usuários dos dois prestadores de serviços utilizam MPI. No uso dos antidemenciais não houve diferenças significativas entre os dois grupos.

No geral, os psicofármacos utilizados pelos usuários do SUS são aqueles de menor custo e são MPI para idosos, enquanto aos usuários do plano de saúde são prescritos medicamentos com menor risco de efeitos indesejáveis. Apesar da pesquisa ter contribuído para evidenciar a problemática que permeia o uso de psicofármacos entre os idosos independentemente da modalidade de atenção à saúde, compreende-se que essa temática carece de estudos mais aprofundados tanto no que se refere ao preparo do prescritor como ao acesso 
às tecnologias de assistência, visto que os usuários do SUS também deveriam se beneficiar de medicamentos com menor risco de efeitos indesejáveis.

Portanto, este estudo mostrou que, embora não existam diferenças significantes entre a quantidade de psicofármacos utilizada por usuários do SUS ao serem comparados com os do PSS, há a sinalização da falta de acesso dos usuários do SUS a medicamentos mais modernos e que causem menos efeitos adversos.

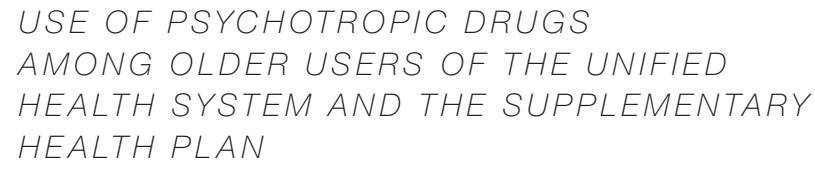

USE OF PSYCHOTROPIC DRUGS

AMONG OLDER USERS OF THE UNIFIED

HEALTH SYSTEM AND THE SUPPLEMENTARY

HEALTH PLAN

\section{abstract}

Objective: To compare the usage of psychoactive drugs among older patients from both the Unified Health System (SUS) and from Supplementary Health Plans (PSS). Methods: A quantitative and retrospective study, making use of two distinct data bases, from which: 239 were older patients of PSSs and 114 were patients of the Family Health Strategy. Statistical analyzes were performed using the Statistical Package for Social Sciences (SPSS) software, version 17.0, for Windows. The inferential analyzes were performed using the Pearson's chi-square test and Fisher's exact test extension. Results: Among SUS users, 53\% were between 60 and 69 years old, and $13 \%$ older than 80 years. Diversely, only $37 \%$ of PSS users were between 60 and 69 years old, 24\% being older than 80 years. In terms of sociodemographic context, SUS users have higher prevalence of black or brown skin, low levels of schooling, and are also part of lower socioeconomic classes. It was found that 68\% of SUS patients and $60 \%$ of PSS patients do not use psychoactive drugs. The most prescribed psychotropic drugs are antidepressants. Amitriptyline, fluoxetine, diazepam, clonazepam and alprazolam, which are lower-cost drugs, but considered inappropriate for older patients, were mainly prescribed to SUS users. Conclusion: SUS and PSS users have different sociodemographic characteristics. SUS patients use larger amounts of potentially inappropriate drugs, while health plan (PSS) users are prescribed more technologically advanced medications with less possibility of undesirable effects. 
AGÊNCIA NACIONAL DE SAÚDE SUPLEMENTAR (ANS). Dados e indicadores do setor. Rio de Janeiro: ANS, 2016. Disponível em: http://www.ans.gov.br/perfil-do-setor/ dados-e-indicadores-do-setor. Acesso em: 5 maio 2016.

AGÊNCIA NACIONAL DE SAÚDE SUPLEMENTAR (ANS). Plano de cuidado para idosos na Saúde Suplementar. Rio de Janeiro: ANS, 2012. Disponível em: http://www.ans. gov.br/images/stories/Materiais_para_pesquisa/Materiais_por_assunto/20121004_ plano_cuidado_idosos.pdf. Acesso em: 8 jul. 2017.

ALVARENGA, Jussara Mendonça et al. Uso de benzodiazepínicos entre idosos: o alívio de "jogar água no fogo", para não pensar e dormir. Revista Brasileira de Geriatria e Gerontologia, Rio de Janeiro, v. 18, n. 2, p. 249-258, 2015.

AMERICAN GERIATRICS SOCIETY 2015 BEERS CRITERIA UPDATE EXPERT PANEL (AGS). American Geriatrics Society 2015 updated beers criteria for potentially inappropriate medication use in older adults. Journal of the American Geriatrics Society, New York, v. 63, n. 11, p. 2227-2246, 2015.

ASSOCIAÇÃO BRASILEIRA DE EMPRESAS DE PESQUISA (ABEP). Critério de Classificação Econômica Brasil. São Paulo: ABEP, 2014. Disponível em: http://www.abep. org/criterio-brasil. Acesso em: 18 maio 2015.

BOTTAN, Gabriela. Determinantes de qualidade de vida de idosos usuários de Centro de Atenção Psicossocial. 2013. 88 f. Dissertação (Mestrado em Enfermagem) - Escola de Enfermagem, Universidade Federal do Rio Grande do Sul, Porto Alegre, 2013.

BRASIL. Ministério da Saúde. Estatuto do idoso. 3. ed. Brasília, DF: Ministério da Saúde, 2013a.

BRASIL. Ministério da Saúde. Secretaria de Atenção à Saúde. Departamento de Atenção Básica. Caderno de atenção domiciliar. Brasília, DF: Ministério da Saúde, 2013b.

BRASIL. Ministério da Saúde. Secretaria de Ciência, Tecnologia e Insumos Estratégicos. Departamento de Assistência Farmacêutica e Insumos Estratégicos. Relação Nacional de Medicamentos Essenciais: RENAME 2014. 9. ed. rev. e atual. Brasília, DF: Ministério da Saúde, 2015.

BUENO, Denise; ALMEIDA, Taiana Trindade de; ROCHA, Bruno Simas. Prevalência de prescrição de medicamentos potencialmente inapropriados para idosos em uma unidade de saúde da família de Porto Alegre/RS. Revista de APS, Juiz de Fora, v. 19, n. 3, p. 370-375, 2016.

CARVALHO, Fabrícia Lopes; CRISTINO, Reviann Rosa; LIMBERGER, Jane Beatriz. Uso racional de medicamentos por pessoas idosas: um enfoque na doença de Alzheimer. Disciplinarum Scientia: Ciências da Saúde, Santa Maria, v. 19, n. 1, p. 99-112, 2018.

CASSONI, Teresa Cristina Jahn et al. Uso de medicamentos potencialmente inapropriados por idosos do Município de São Paulo, Brasil: Estudo SABE. Cadernos de Saúde Pública, Rio de Janeiro, v. 30, n. 8, p. 1708-1720, 2014. 
CLEMENTE, Adauto Silva; LOYOLA FILHO, Antônio Ignácio; FIRMO, Josélia Oliveira Araújo. Concepções sobre transtornos mentais e seu tratamento entre idosos atendidos em um serviço público de saúde mental.Cadernos de Saúde Pública, Rio de Janeiro, v. 27, n. 3, p. 555-564, 2011.

COLET, Christiane de Fátima; BORGES, Paulo Eduardo Mayorga; AMADOR, Tânia Alves. Perfil de gastos com medicamentos entre idosos em diferentes grupos socioeconômicos. Revista Brasileira de Geriatria e Gerontologia, Rio de Janeiro, v. 19, n. 4, p. 591-601, 2016 .

COSTA, Roberta Dorneles Ferreira et al. Aquisição de medicamentos para a Doença de Alzheimer no Brasil: uma análise no sistema federal de compras, 2008 a 2013. Ciência \& Saúde Coletiva, Rio de Janeiro, v. 20, n. 12, p. 3827-3838, 2015.

DILÉLIO, Alitéia Santiago et al. Padrões de utilização de atendimento médico-ambulatorial no Brasil entre usuários do Sistema Único de Saúde, da saúde suplementar e de serviços privados. Cadernos de Saúde Pública, Rio de Janeiro, v. 30, n. 12, p. 2594-2606, 2014

FICK, Donna et al. Updating the Beers criteria for potentially inappropriate medication use in older adults: results of a US consensus panel of experts. Archives of Internal Medicine, Chicago, v. 163, n. 22, p. 2716-2724, 2003.

GORZONI, Milton Luiz; FABBRI, Renato Moraes Alves; PIRES, Sueli Luciano. Medicamentos potencialmente inapropriados para idosos. Revista da Associação Médica Brasileira, São Paulo, v. 58, n. 4, p. 442-446, 2012.

HOLT, Stefanie; SCHMIEDL, Sven; THÜRMANN, Petra A. Potentially inappropriate medications in the elderly: The PRISCUS List. Deutsches Ärzteblatt International, Alemanha, v. 107, n. 31-32, p. 543-551, 2010.

INSTITUTO BRASILEIRO DE GEOGRAFIA E ESTATÍSTICA (IBGE). Censo demográfico 2010: características gerais da população, religião e pessoas com deficiência. Rio de Janeiro: IBGE, 2012. Disponível em: http://biblioteca.ibge.gov.br/visualizacao/periodicos/94/cd_2010_religiao_deficiencia.pdf. Acesso em: 3 mar. 2017.

INSTITUTO BRASILEIRO DE GEOGRAFIA E ESTATÍSTICA (IBGE). Cidades@: São Paulo: Marília. Rio de Janeiro: IBGE, 2017. Disponível em: http://cidades.ibge.gov.br/ painel/painel.php?lang=\&codmun=352900\&search=|marilia. Acesso em: 3 mar. 2017.

INSTITUTO BRASILEIRO DE GEOGRAFIA E ESTATÍSTICA (IBGE). Síntese de indicadores sociais: uma análise das condições de vida da população brasileira - 2013. Rio de Janeiro: IBGE, 2013. Disponível em: http://biblioteca.ibge.gov.br/visualizacao/livros/ liv66777.pdf. Acesso em: 3 mar. 2017.

MARIN, Maria José Sanches et al. Caracterização do uso de medicamentos entre idosos de uma unidade do Programa Saúde da Família. Cadernos de Saúde Pública, Rio de Janeiro, v. 24, n. 7, p. 1545-1555, 2008.

MUNIZ, Elaine Cristina Salzedas et al. Análise do uso de medicamentos por idosos usuários de plano de saúde suplementar. Revista Brasileira de Geriatria e Gerontologia, Rio de Janeiro, v. 20, n. 3, p. 375-387, 2017.

NOIA, Aparecida Santos et al. Fatores associados ao uso de psicotrópicos por idosos residentes no Município de São Paulo. Revista da Escola de Enfermagem da USP, São Paulo, v. 46, número especial, p. 38-43, 2012.

OLIVEIRA, Amanda Maria Souza de; MENEZES, Tânia Maria de Oliva. A enfermeira no cuidado ao idoso na Estratégia Saúde da Família: sentidos do vivido. Revista de Enfermagem da UERJ, Rio de Janeiro, v. 22, n. 4, p. 513-518, 2014.

OLIVEIRA, Gabriella Stravini de; CERDEIRA, Cláudio Daniel; BARROS, Gérsika Bitencourt Santos. Estudo epidemiológico da prescrição de medicamentos potencialmente inapropriados para idosos no município de Alfenas/Minas Gerais. Revista da Universidade vale do Rio Verde, Três Corações, v. 15, n. 1, p. 508-515, 2017. 
ONOFRI JÚNIOR, Venício Aurélio; MARTINS, Vinícius Spazzapan; MARIN, Maria José Sanches. Atenção à saúde do idoso na Estratégia Saúde da Família e a presença de transtornos mentais comuns. Revista Brasileira de Geriatria e Gerontologia, Rio de Janeiro, v. 19, n. 1, p. 21-33, 2016.

PAULA, Tatiana Cruz de; BOCHNER, Rosany; MONTILLA, Dalia Elena Romero. Análise clínica e epidemiológica das internações hospitalares de idosos decorrentes de intoxicações e efeitos adversos de medicamentos, Brasil, de 2004 a 2008. Revista Brasileira de Epidemiologia, São Paulo, v. 15, n. 4, p. 828-844, 2012.

$\mathrm{ROCHA}$, Saulo Vasconcelos et al. Prevalência de transtornos mentais comuns entre idosos residentes em município do Nordeste do Brasil. Revista de Salud Pública, Bogotá, v. 14, n. 4, p. 620-629, 2012.

SANTOS, Thalyta Renata Araújo et al. Consumo de medicamentos por idosos, Goiânia, Brasil. Revista de Saúde Pública, São Paulo, v. 47, n. 1, p. 94-103, 2013.

SILVA, Jerto Cardoso da; HERZOG, Lísia Mânica. Psicofármacos e psicoterapia com idosos. Psicologia \& Sociedade, Belo Horizonte, v. 27, n. 2, p. 438-448, 2015.

TAVARES, Darlene Mara dos Santos et al. Qualidade de vida e adesão ao tratamento farmacológico entre idosos hipertensos. Revista Brasileira de Enfermagem, Brasília, DF, v. 69, n. 1, p. 134-141, 2016.

THORLUND, Kristian et al. Comparative efficacy and safety of selective serotonin reuptake inhibitors and serotonin-norepinephrine reuptake inhibitors in older adults: a network meta-analysis. Journal of the American Geriatrics Society, New York, v. 63, n. 5, p. 1002-1009, 2015.

VIDAL, Carlos Eduardo Leal et al. Transtornos mentais comuns e uso de psicofármacos em mulheres. Cadernos Saúde Coletiva, Rio de Janeiro, v. 21, n. 4, p. 457-464, 2013. 